\title{
ACTIVIDADES GENERALES DE LA DIPUTACION PROVINCIAL DE CADIZ EN 1979
}

\author{
353.2.075.1 (46. Cádiz)
}

por

\section{Antonio Rodríguez Socorro}

Jefe de la Sección de Estadística del Instituto de Estudios de Administración Local

SUMARIO: I. INTRODUCCION.-II. CARACTERISTICAS DE LA PROVINCIA: 1. TERRITORIO Y POBLACIÓN. 2. COMUNICACIONES. 3. TURISMO.-III. ORGANIZACION, FUNCIONAMIENTO Y ACTIVIDADES GENERALES DE LA DIPUTACION PROVINCIAL: 1. LA CORPORACIÓN PROVINCIAL. 2. ACTIVIDAD CORPORATIVA. 3. ORGANIZACIÓN ADMINISTRATIVA Y PERSONAL.-IV. SERVICIOS DE COMPETENCIA PROVINCIAL: 1. CONSTRUCCIÓN Y CONSERVACION DE VIAS PROVINCIALES. 2. HOSPITAL "MORA», PROVINCIAL. 3. Hospital pSiquiátrico «Nuestra SeÑora de la PAZ». 4. AmbulatoRIOS PSIQUítricicos. 5. Hogar PROVINCIAL «NUESTRA SEÑORA DE los Dolores", en Jerez. 6. Centros concertados. 7. Hogar iNFANTIL "MigUEL ARAMBURU E INDA». 8. INSTITUCIÓN PROVINCIAL gaditana. 9. Colegio "Carlos Marfa R. DE Valcárcel». 10. Residencia provincial de ancianos (Puerto de Santa Marfa). 11. CenTRO REGIONAL DE EDUCACIÓN ESPECIAL POLIVALENTE (SUBNORMALeS). 12. Collegio Para SORDOMUdos. 13. Colegio Universitario gaditano. 14. Centro regional de la Universidad Nacional a Distancia. 15. Instituto de Estudios Gaditanos. 16. AyUdas a MaNifESTaciones CUlturales. 17. CENTRO COORDiNador DE BIBlioteCAS de CÁdz. 18. Centros experimentales: A) Centro Experi- 
mental Agrícola-Ganadero. B) Centro Experimental de Cultivos. C) Escuela de Capataces Agrícolas. 19. INSTALACIONES DEPORTIvas. 20. Hostal «Las Truchas». 21. Servicio Provincial contra Incendios. 22. Parque Movil. 23. Economato Provincial. 24. BieNeS MUEbles de la Diputación Provincial. 25. Viviendas de la DiPUTACIÓN PROVINCIAL.-V. COOPERACION PROVINCIAL A LOS SERVICIOS PROVINCIALES: 1. Planes DE COOPERACIÓN. 2. Plan PRovincial de obras y SERvicios de 1979. 3. AyUda técNica a los Municipios. 4. Caja Provincial de Crédito MuniciPAL.-VI. AMPLIACION DEL ABASTECIMIENTO DE AGUAS A LA ZONA DE LA BAHIA GADITANA.-VII. HACIENDA PROVINCIAL: 1. PRESUPUESTO ORDINARIO DE GASTOS, INGRESOS Y LIQUIDACIÓN DEL AÑo 1979: A) Presupuesto gastos (1979) (segundo semestre). B) Presupuesto ingresos (1979) (segundo semestre). C) Liquidación del presupuesto ordinario 1979. 2. PRESUPUESTo DE GaSTOS E INGRESOS Y LIQUIDACIÓN DEL SERVICIO DE RECAUdACIÓN DE Contribuciones E Impuestos del Estado (EJERcicio 1979): A) Presupuesto de gastos. B) Presupuesto de ingresos. C) Liquidación del presupuesto especial de contribuciones 1979. 3. PRESUPUESTOS EXTRAORDINARIOS (AL 30 DE DICIEMBRE DE 1979). 4. INVENTARIO DE BIENES PROPIEDAD DE LA DiPUTACIÓN PROVINCIAL.VIII. EPILOGO.

\section{INTRODUCCION}

Se trata de comentar, fundamentalmente, realizaciones y valorar las concesiones de la Diputación Provincial para la organización, funcionamiento y actividades generales de los servicios de competencia provincial.

La Memoria anual, redactada por la Secretaría y enviada al Instituto de Estudios de Administración Local con fecha 25 de junio de 1980, cumple lo dispuesto en el número 6 del artículo 144 del Reglamento de Funcionarios de Administración Local de 30 de mayo de 1952 y Circular de la Dirección General de Administración Local de 25 de mayo de 1951, completada con otra del propio Organismo de 13 de mayo de 1953, nos informa de todo ello. 


\section{CARACTERISTICAS DE LA PROVINCIA}

\section{TERRITORIO Y POBLACIÓN}

La Provincia de Cádiz se halla situada en el extremo meridional de la Península Ibérica, y pertenece a la misma el término municipal de Tarifa, punto más alejado del centro geográfico de Europa.

Está constituida por 42 Municipios y 12 Partidos judiciales. La Provincia tiene unos 7.388 kilómetros cuadrados y ocupa el lugar trigésimo sexto en el orden de extensión de las Provincias españolas. La extensión media de los Municipios de la Provincia es de 178,83 kilómetros cuadrados. El término municipal de menos extensión es el de la capital con 16,68 kilómetros cuadrados.

La Provincia presenta notables contrastes orográficamente $y$, por ello, diversas zonas climatológicas y Comarcas naturales: doce Municipios serranos, diez Municipios de campiña, diez Municipios en el litoral y el Campo de Gibraltar con siete Municipios.

Respecto de la población, el padrón oficial de 1975 no ha sufrido variación al 31 de diciembre de 1978.

\section{COMUNICACIONES}

En la red de carreteras y caminos de la Provincia se distinguen las pertenecientes al Estado, las dependientes de la Diputación Provincial y las de peaje.

En el primer grupo están las nacionales (N-340, 342 y IV), las comarcales (C-329, 333, 341, 343, 344, 346, 440 y 441) y las locales (CA-201, 202, 203, 204, 211, 212, 213, 214, 221, 222, 223, 224, 231, 232, 233, 234, 401, 402, 403, 413, 414, 421, 422, 441, 454, 501, 502, 503, $504,511,512,513,514,521,522,523,524,531,532,533,534,541$, $601,602,603,604,611,612,613,614$ у 624).

Está en funcionamiento la autopista de peaje Cádiz-Sevilla, con una longitud de 93,5 kilómetros.

Los caminos vecinales figuran en los servicios de la competencia de la Diputación Provincial.

La red ferroviaria de la Provincia no ha sufrido variación alguna en los últimos años.

La Provincia cuenta con los siguientes puertos, por orden alfabético: Algeciras, Barbate, Cádiz, Puerto Real, Puerto de Santa María, Rota, San Fernando, Sanlúcar de Barrameda y Tarifa. 
Los faros y señales marítimas de la Provincia están situados en Cádiz, Rota, Chipiona, Trafalgar, Bonanza, Sancti Petri, Punta de Carnero, San Jerónimo, Tarifa, Barbate, Isla Verde y Punta Paloma.

El aeródromo de la Provincia está en Jerez.

\section{TURISMO}

Se pueden considerar cinco rutas turísticas, que por sus adjetivos señalan sus principales características:

- La ruta del vino (Jerez de la Frontera: vinos, caballos, ferias..., y las iglesias gótica de San Miguel y mudéjar de San Dionisio, Museo Arqueológico, Biblioteca Municipal...; Rota, Trebujena...).

- La ruta atlántica (Cádiz con sus monumentos trimilenarios, Puerto Real, Chipiona...).

- La ruta serrana (de Algodonales a la árabe Olvera: una calle, una iglesia, un castillo...; Ubrique, El Bosque...).

- La ruta del toro (saliendo de Jerez se atraviesan las dehesas de Martelilla y Fuenterrey, viendo pastar a ganaderías famosas; Medina Sidonia, Algeciras, San Roque...).

- La ruta de los pueblos blancos (carretera de Jerez a Arcos de la Frontera, «uno de los pueblos más bonitos de España», con palabras de Azorín, y repetido gratamente en tantas películas españolas; Ronda...).

\section{ORGANIZACION, FUNCIONAMIENTO Y ACTIVIDADES GENERALES DE LA DIPUTACION PROVINCIAL}

\section{La CORPORACIÓN PROVINCIAL}

Fue presidente de la excelentísima Diputación Provincial, desde el 4 de noviembre de 1969, el excelentísimo señor don Antonio Barbadillo y García de Velasco, con una reelección, hasta el 26 de abril de 1979 que cesó, y estaba constituida por 20 miembros, de hecho 15, incluido el excelentísimo señor presidente.

La nueva Corporación tiene por presidente a don Gervasio Hernández Palomeque y está constituida por 27 miembros, incluido el presidente, y tomó posesión el 26 de abril de 1979. 
La Corporación provincial, en sesión celebrada el día 10 de mayo de 1979, acordó la constitución de las Comisiones informativas siguientes: Gobierno, Hacienda y Economía y de Presupuestos y Control del Gasto, Régimen Interior y Personal, Sanidad, Acción Social y Promoción Ciudadana, Urbanismo y Vivienda, Cultura, Información y Publicaciones, Educación Física y Deportes, Planes Provinciales, Agricultura y Ganadería, Industria y Energía, Obras Públicas y Paro Obrero, Junta Central de Compras, Fomento del Turismo y Caja Provincial de Crédito Municipal.

El número de resoluciones de la Presidencia durante el ejercicio de 1979 fue de 1.828, y el número de sesiones celebradas por el Pleno en el mismo ejercicio fueron 21.

\section{ACTIVIDAD CORPORATIVA}

Ha sobresalido la visita al excelentísimo señor presidente del excelentísimo señor Gobernador civil de la Provincia, y las visitas y reuniones de cortesía de la Corporación a las autoridades de la Provincia.

\section{ORganización aDMinistrativa y PERSONAL}

La organización administrativa de la Diputación Provincial de Cádiz está constituida fundamentalmente por tres grandes dependencias: Secretaría, Intervención y Depositaría, además de Asesoría Jurídica, Secretaría Particular, Mayordomía y Secciones de Arquitectura, Obras y Vías e Ingeniería Industrial, y diversos organismos de tipo hospital, residencial-psiquiátrico, hogar infantil, colegios, parque móvil, ciudad deportiva, residencia de ancianos, centros de educación especial, etc.

El personal que atiende esos servicios es: de plantilla, 687; personal laboral, 678, y personal contratado, 27 (según el art. 8. del Reglamento de Funcionarios).

\section{SERVICIOS DE COMPETENCIA PROVINCIAL}

1. CONSTRUCCIÓN Y CONSERVACIÓN DE VIAS PROVINCIALES

El resumen económico de este capítulo puede expresarse así: 


\begin{tabular}{ccc}
$\begin{array}{c}\text { Conservación } \\
\text { Pesetas }\end{array}$ & $\begin{array}{c}\text { Modernización } \\
\text { Pesetas }\end{array}$ & $\begin{array}{c}\text { Otras obras } \\
\text { realizadas } \\
-\end{array}$ \\
\cline { 2 - 3 } 13.413 .031 & 21.668 .611 & $\frac{\text { Pesetas }}{203.764 .400}$ \\
\hline
\end{tabular}

2. HoSPITAL «MORA», PROVINCIAL

Los ingresados de beneficiencia en este hospital durante el año 1979 fueron 955 y 18.766 estancias, y el total de ingresados, 5.547 enfermos y 85.459 estancias.

El resumen económico de este capítulo se puede expresar así:

\begin{tabular}{|c|c|c|c|}
\hline $\begin{array}{c}\text { Ingresos } \\
- \\
\text { Pesetas }\end{array}$ & $\begin{array}{c}\text { Gastos } \\
\text { Pesetas }\end{array}$ & $\begin{array}{c}\text { Aportación } \\
\text { de la Diputación } \\
\text { Provincial } \\
- \\
\text { Pesetas }\end{array}$ & $\begin{array}{c}\text { Obras } \\
y \\
\text { adquisiciones } \\
- \\
\text { Pesetas }\end{array}$ \\
\hline 341.131 .315 & 563.072 .697 & 221.941 .382 & 19.683 .946 \\
\hline
\end{tabular}

\section{Hospital psiquiatrico «Nuestra SeÑora de la PaZ»}

Este hospital está ubicado en la finca «El Madrugador», del término municipal de Puerto de Santa María, con una superficie construida de 23.348 metros cuadrados. La recepción de enfermos se efectúa a diario y a cualquier hora, ya que cuenta con servicio de urgencia completo: médicos, ayudantes técnicos sanitarios, cuidadores y ambulancia.

El resumen económico de este capítulo puede expresarse así:

\begin{tabular}{|c|c|c|c|}
\hline $\begin{array}{c}\text { Ingresos } \\
\text { Pesetas }\end{array}$ & $\begin{array}{c}\text { Gastos } \\
\text { Pesetas }\end{array}$ & $\begin{array}{c}\text { Aportación } \\
\text { de la Diputación } \\
\text { Provincial } \\
\text { Pesetas }\end{array}$ & $\begin{array}{c}\text { Obras } \\
y \\
\text { adquisiciones } \\
- \\
\text { Pesetas }\end{array}$ \\
\hline 19.072 .419 & 304.869 .183 & 285.796 .764 & 9.214 .335 \\
\hline
\end{tabular}




\section{AmbULATORIOS PSIQUiÁtricos}

Existen dos: uno central, en Cádiz, que atiende a 19 Municipios, y otro en Algeciras, que atiende toda la zona del Campo de Gibraltar, con siete Municipios.

5. Hogar provincial «Nuestra SeÑora de los Dolores», en Jerez

Este Hogar provincial da albergue a mujeres enfermas y subnormales, imposibilitadas y de difícil adaptación familiar.

Los gastos de sostenimiento fueron de 21.502.275 pesetas, y las obras y adquisiciones del primer establecimiento fueron de pesetas 741.268.

\section{Centros concertados}

Además de la Institución Provincial Gaditana, Institución «Carlos María R. de Valcárcel», Hogar Infantil, Hogar «Nuestra Señora de los Dolores" y Residencia de Ancianos, todos ellos centros propios de la excelentísima Diputación Provincial, la Diputación tiene establecidos conciertos con siete centros, con una capacidad de 358 plazas, y el presupuesto es de 17.664 .520 pesetas.

\section{Hogar INFANTIL «Miguel ARamburu E INDA»}

Está adecuado para niños de cero a siete años y niñas de cero a ocho años. Los gastos de sostenimiento fueron de 115.492 .938 pesetas, y las obras y adquisiciones fueron por valor de 4.513.017 pesetas.

\section{Instituctón Provincial Gaditana}

Esta Institución lleva a cabo la educación e instrucción de las niñas y jóvenes tuteladas por la Corporación provincial, en cumplimiento de la misión que le asigna la Ley de Administración Local y la propia del centro de enseñanza, que con precios módicos 
contribuyen a paliar la falta de centros de enseñanza femeninos en la capital y, a la vez, permite internados de alumnas de localidades de la Provincia. En la primera etapa de EGB (1. a $\left.5 .^{\circ}\right)$ sostiene 25 secciones; en la segunda etapa $\left(6 .^{\circ}\right.$ a $\left.8^{\circ}\right), 15$ secciones; en Formación Profesional Industrial, nuevo plan, primer grado, sostiene cuatro secciones, y segundo grado de enseñanza especializada, tres secciones; todo ello desarrollado con 134 alumnos de Preescolar, 1.721 de EGB y 251 de FPI.

El resumen económico de este capítulo puede expresarse así:

\begin{tabular}{|c|c|c|c|}
\hline $\begin{array}{c}\text { Ingresos } \\
\text { Pesetas }\end{array}$ & $\begin{array}{c}\text { Gastos } \\
- \\
\text { Pesetas }\end{array}$ & $\begin{array}{c}\text { Aportación } \\
\text { de la Diputación } \\
\text { Provincial } \\
- \\
\text { Pesetas }\end{array}$ & $\begin{array}{c}\text { Obras } \\
y \\
\text { adquisiciones } \\
- \\
\text { Pesetas }\end{array}$ \\
\hline 17.212 .241 & 124.680 .441 & 107.468 .200 & 1.582 .832 \\
\hline
\end{tabular}

\section{Colegto «Carlos Marfa R. de Valcárcel»}

Las actividades de este colegio han sido las normales, siguiendo el proceso de reestructuración orgánico-funcional para lograr una asistencia, formación y enseñanza de la más alta calidad.

El internado se compone de 360 alumnos, de los cuales 300 son de beneficencia y 60 becarios del PIO, pero asisten muchos más, externos, ya que los alumnos son 1.303, y son atendidos por 20 de personal religioso, 43 de personal docente y 112 de personal no docente.

El resumen económico de este capítulo puede expresarse así:

\begin{tabular}{|c|c|c|c|}
\hline $\begin{array}{c}\text { Ingresos } \\
\text { Pesetas }\end{array}$ & $\begin{array}{c}\text { Gastos } \\
\text { Pesetas }\end{array}$ & $\begin{array}{c}\text { Aportación } \\
\text { de la Diputación } \\
\text { Provincial } \\
- \\
\text { Pesetas }\end{array}$ & $\begin{array}{c}\text { Obras } \\
y \\
\text { adquisiciones } \\
- \\
\text { Pesetas }\end{array}$ \\
\hline 17.569 .096 & 117.019 .766 & 99.450 .670 & 2.113 .950 \\
\hline
\end{tabular}


10. Residencia Provincial de Ancianos (Puerto de Santa Maria)

Esta residencia ha venido desempeñando todas las funciones asistenciales para las que fue creada, exagerando, si cabe, las medidas para que las molestias para el anciano, en el momento del ingreso y su instalación definitiva, sean mínimas.

El resumen económico de este capítulo puede expresarse así:

\begin{tabular}{|c|c|c|c|}
\hline $\begin{array}{c}\text { Ingresos } \\
\text { Pesetas }\end{array}$ & $\begin{array}{c}\text { Gastos } \\
\text { Pesetas }\end{array}$ & $\begin{array}{c}\text { Aportación } \\
\text { de la Diputación } \\
\text { Provincial } \\
- \\
\text { Pesetas }\end{array}$ & $\begin{array}{c}\text { Obras } \\
y \\
\text { adquisiciones } \\
- \\
\text { Pesetas }\end{array}$ \\
\hline 15.602 .350 & 47.680 .400 & 32.078 .050 & 2.900 .099 \\
\hline
\end{tabular}

\section{Centro Regional de Educación Especial Polivalente (SubNoRmales)}

En el año 1979 ha estado este centro, situado en Puerto de Santa María, organizado para atender 180 alumnos (140 internos y 40 mediopensionistas): a nivel preescolar, ocho aulas (12 alumnos por aula); EGB, cursos $1 .^{\circ}, 2 .^{\circ}$ y $3 .^{\circ}$, cuatro aulas (13 y 14 alumnos por aula); un aula de laborterapia con 12 alumnos, un grupo de talleres (un aula con 14 alumnos) y un aula de grupos maternales con cuatro alumnos.

El resumen económico de este capítulo se puede expresar así:

\begin{tabular}{|c|c|c|c|}
\hline $\begin{array}{c}\text { Ingresos } \\
\text { Pesetas }\end{array}$ & $\begin{array}{c}\text { Gastos } \\
\text { Pesetas }\end{array}$ & $\begin{array}{c}\text { Aportación } \\
\text { de la Diputación } \\
\text { Provincial } \\
- \\
\text { Pesetas }\end{array}$ & $\begin{array}{c}\text { Obras } \\
y \\
\text { adquisiciones } \\
- \\
\text { Pesetas }\end{array}$ \\
\hline 817.572 & 81.886 .070 & 81.068 .498 & 2.937 .817 \\
\hline
\end{tabular}

\section{COLEGIO PARA SORDOMUDOS}

En este centro, situado en Jerez, durante el curso 1978-79 el número de alumnos matriculados en el centro fue de 90 , con eda- 
des comprendidas entre los tres y diecisiete años, en 12 unidades, y en el curso $1979-80$ son 100 alumnos.

El resumen económico de este capítulo puede expresarse así:

\begin{tabular}{|c|c|c|}
\hline $\begin{array}{c}\text { Ingresos } \\
\text { Pesetas }\end{array}$ & $\begin{array}{c}\text { Gastos } \\
\text { Pesetas }\end{array}$ & $\begin{array}{c}\text { Aportación } \\
\text { de la Diputación } \\
\text { Provincial } \\
- \\
\text { Pesetas }\end{array}$ \\
\hline 1.170 .000 & 19.190 .399 & 18.820 .399 \\
\hline
\end{tabular}

\section{Colegio Universitario Gaditano}

El Patronato de Promoción Universitaria de la Provincia de Cádiz nació como órgano promotor y creador del Colegio Universitario Gaditano, que ha de servir de base a la futura Universidad de Cádiz.

En la sesión del 29 de abril de 1969 celebrada en la Diputación Provincial se acordó llevar a cabo la creación de un Colegio Universitario adscrito a la Universidad de Sevilla (de acuerdo con el Decreto 452/1969, de 27 de marzo, y el artículo 84 del Reglamento de Servicios de las Corporaciones locales).

Fue autorizado por el Ministerio de Educación y Ciencia con fecha 6 de agosto de 1969 para impartir en él las enseñanzas correspondientes al primer curso de los estudios comunes de la Facultad de Filosofía y Letras y curso selectivo de Ciencias. Por Decreto $2745 / 1971$, de 14 de octubre, se reconoció al Colegio Universitario adscrito a la Universidad de Sevilla y autorizándole a impartir el primer ciclo de los estudios de Filosofía y Letras y Ciencias.

En el curso 1979-80, el presupuesto de gastos es de 47.181.307 pesetas y el coste por alumno y curso resulta a 122.618 pesetas.

\section{Centro Regional de la Universidad Nacional a Distancia}

La UNED fue creada por Decreto 2310/1972, de 18 de agosto, y en enero de 1974 empieza a funcionar, recibiendo alumnos, aparte de los de la Provincia, de las Provincias de Córdoba, Sevilla, Huelva y de la ciudad de Ceuta. 
En el año 1979 tuvo 2.902 alumnos y en 1980 son 2.509, desglosados en los estudios de Derecho, Ciencias de la Educación, Geografía e Historia, Filosofía, Psicología, Ciencias Económicas y Empresariales, Ciencias Físicas, Ciencias Químicas y Ciencias Matemáticas, Ingeniería Industrial, Curso de Acceso, C. A. Profesores Mercantiles y C. Adapt. Peritos.

La Diputación Provincial aportó en 1979 la cantidad de pesetas 27.324.756.

\section{Instituto DE Estudios Gaditanos}

Fue creado este centro en 1978 y ha realizado una labor cultural extensa y de alto nivel, con publicaciones importantes, bienales de artes plásticas, de pintura, dibujo, grabado; colaboraciones con la Feria del Libro...

Los gastos han sido de 2.779 .513 pesetas.

\section{AYUDAS A MANIFESTACIONES CULTURALES}

Se han concedido por parte de la Diputación Provincial en el año 1978 muchas ayudas económicas a entidades y centros que las solicitaron.

El total de gastos fue de 7.336 .715 pesetas.

\section{Centro Coordinador de Bibliotecas de Cadiz}

El Patronato del Centro ha llevado a cabo una excelente labor con un presupuesto de gastos en 1979, a cargo de la Diputación Provincial, de 2.396 .025 pesetas.

\section{Centros experimentales}

\section{A) Centro Experimental Agricola-Ganadero}

Este Centro, situado en Jerez de la Frontera, se creó con los fines siguientes: experimentación en agricultura, variedades de fertilización, cultivos, ensayos de ganadería, rendimientos en las explotaciones, fomento de forrajes y colaboraciones con otras Diputaciones, organismos y agricultores. 
El régimen económico de este capítulo se puede expresar así:

\begin{tabular}{|c|c|c|c|}
\hline $\begin{array}{c}\text { Ingresos } \\
\text { Pesetas }\end{array}$ & $\begin{array}{c}\text { Gastos } \\
\text { Pesetas }\end{array}$ & $\begin{array}{c}\text { Aportación } \\
\text { de la Diputación } \\
\text { Provincial } \\
- \\
\text { Pesetas }\end{array}$ & $\begin{array}{c}\text { Obras } \\
y \\
\text { adquisiciones } \\
\text { Pesetas }\end{array}$ \\
\hline 7.116 .475 & 32.301 .954 & 25.185 .479 & 300.000 \\
\hline
\end{tabular}

\section{B) Centro Experimental de Cultivos}

Este Centro, situado en Chipiona, tiene como fines: experimentación hortícola sobre cultivos en campos e invernaderos, colaboraciones y divulgación de resultados.

El régimen económico de este capítulo se puede expresar así:

\begin{tabular}{ccc}
$\begin{array}{c}\text { Ingresos } \\
\text { Pesetas }\end{array}$ & Gastos & $\begin{array}{c}\text { Aportación } \\
\text { de la Diputación } \\
\text { Provincial } \\
255.144\end{array}$ \\
& $\begin{array}{c}\text { Pesetas } \\
\text { Pesetas }\end{array}$ \\
\hline
\end{tabular}

\section{C) Escuela de Capataces Agrícolas}

Este centro está situado en Chipiona, y la especialidad que allí se practica y desarrolla es la de Horticultura en cursillos intensivos.

\section{INSTALACIONES DEPORTTVAS}

Figuran las siguientes:

A) Instalaciones incluidas en el convenio con el Consejo Superior de Deportes, con un presupuesto de gastos de 21.468 .000 pesetas.

B) Obras propias de la Diputación en instalaciones, con un presupuesto de gastos de 4.111.701 pesetas.

C) Pabellón Deportivo Provincial de Cádiz, con la aportación de la Diputación de 10.957.288 pesetas. 
D) Ciudad Deportiva del Puerto de Santa María, con la aportación de la Diputación de 5.189.224 pesetas.

E) Complejo Polideportivo de Sanlúcar de Barrameda, con la aportación de la Diputación de 2.439 .615 pesetas.

\section{Hostal «LAS Truchas»}

Fue construido por iniciativa de la Diputación Provincial para promocionar las rutas turísticas de la sierra gaditana.

El resultado económico de este capítulo se puede expresar así:

\begin{tabular}{|c|c|c|c|}
\hline $\begin{array}{c}\text { Ingresos } \\
\text { Pesetas }\end{array}$ & $\frac{\text { Gastos }}{\text { Pesetas }}$ & $\begin{array}{c}\text { Aportación } \\
\text { de la Diputación } \\
\text { Provincial } \\
- \\
\text { Pesetas }\end{array}$ & $\begin{array}{c}\text { Obras } \\
y \\
\text { adquisiciones } \\
- \\
\text { Pesetas }\end{array}$ \\
\hline 13.555 .058 & 20.632 .991 & 7.077 .933 & 1.196 .323 \\
\hline
\end{tabular}

También se programó para atracción turística de la sierra la piscifactoría en El Bosque.

\section{Servicio Provincial contra Incendios}

Este Servicio consta de nueve Parques comarcales. Durante el año 1979 se realizaron 72 intervenciones, 31 de gran importancia y nueve falsas alarmas.

El resumen económico de este capítulo se expresa así:

\begin{tabular}{|c|c|c|c|}
\hline $\begin{array}{c}\text { Ingresos } \\
\text { Pesetas }\end{array}$ & $\begin{array}{c}\text { Gastos } \\
\text { Pesetas }\end{array}$ & $\begin{array}{c}\text { Aportación } \\
\text { de la Diputación } \\
\text { Provincial } \\
- \\
\text { Pesetas }\end{array}$ & $\begin{array}{c}\text { Obras } \\
y \\
\text { adquisiciones } \\
- \\
\text { Pesetas }\end{array}$ \\
\hline 102.581 & 1.266 .511 & 1.163 .930 & 642.000 \\
\hline
\end{tabular}

\section{PARQUe MÓvII}

Este Servicio está constituido por diverso material móvil, y tiene por resumen económico: gastos de sostenimiento, 23.888.696 pesetas, y para obras y adquisiciones, 1.390.297 pesetas. 


\section{Economato Provincial}

Este Servicio ha quedado reducido a un concierto con tres autoservicios de la plaza, en los cuales los beneficiarios interesados adquieren enseres y efectos mediante vales facilitados por la Depositaría de Fondos Provinciales.

El resumen económico de este capítulo se expresa así:

\begin{tabular}{|c|c|c|c|}
\hline $\begin{array}{c}\text { Ingresos } \\
\text { Pesetas }\end{array}$ & $\begin{array}{c}\text { Pagos } \\
\text { Pesetas }\end{array}$ & $\begin{array}{c}\text { Pendiente de cobro } \\
\text { Pesetas }\end{array}$ & $\begin{array}{c}\text { Pendiente de pago } \\
\text { Pesetas }\end{array}$ \\
\hline 7.112 .492 & 13.861 .421 & 6.539 .759 & 1.452 .800 \\
\hline
\end{tabular}

24. Bienes muebles de la Diputación Provincial

Estos bienes llevan consigo, por adquisición y conservación, los gastos de 49.048 .417 pesetas.

25. Viviendas de la Diputación Provincial

El resumen económico de este capítulo se puede expresar así:

\begin{tabular}{|c|c|c|}
\hline & $\begin{array}{l}\text { Número } \\
\text { de } \\
\text { viviendas }\end{array}$ & $\begin{array}{l}\text { Valor } \\
\frac{-}{\text { Pesetas }}\end{array}$ \\
\hline $\begin{array}{llllll}\text { Terminadas } & \ldots & \ldots & \ldots & \ldots & \ldots \\
\text { En construccion } & \ldots & \ldots & \ldots & \ldots \\
\text { Total certificaciones } & \ldots & \ldots & \ldots\end{array}$ & $\begin{array}{l}408 \\
144 \\
-\end{array}$ & $\begin{array}{l}316.514 .936 \\
210.902 .142 \\
258.428 .741\end{array}$ \\
\hline
\end{tabular}

\section{COOPERACION PROVINCIAL A LOS SERVICIOS MUNICIPALES}

1. Planes de cooperación

La cooperación provincial a obras y servicios se efectuaba con normalidad mediante la confección de Planes bienales que, previos los trámites de aprobación y exposición pública por la Diputación, eran aprobados por el Ministerio de la Gobernación (o Interior).

Conforme a las instrucciones dadas últimamente, confeccionaron, en coordinación con los Planes bienales y de la Comisión de 
la Colaboración del Estado con las Corporaciones Locales, los Planes de cooperación.

La necesidad de dar cumplimiento a lo dispuesto en el artículo 49 de la Ley 47/1975, de 30 de diciembre, de los Presupuestos Generales del Estado para 1976, así como la adecuación del régimen de Planes provinciales, ha impuesto la regulación de todo lo concerniente al nuevo sistema de los Planes provinciales de obras y servicios municipales. Y por ello se dictó el Real Decreto 1087/ 1976, de 23 de abril, sobre la regulación del sistema de Planes provinciales, y se establece la elaboración de un Plan único de obras $y$ servicios que abarque todas las realizaciones provinciales y cuyo estudio, elaboración y ejecución se encomienda a las Diputaciones. Con ello desaparece la dualidad de Planes provinciales mantenida con anterioridad, con una existencia de un Plan de la Comisión Provincial de Servicios Técnicos.

El Plan de cooperación de 1978 fue elaborado de conformidad con lo previsto en el Real Decreto 588/1978, de 17 de febrero, y normas complementarias aprobadas por el Real Decreto 1179/1978, de 15 de julio.

\section{Plan PRovincial de obras y SERVictos DE 1979}

La constitución de las nuevas Corporaciones democráticas demoró la redacción del Plan de obras y servicios para el año 1979, y, tras su aprobación inicial y exposición pública, se aprobó definitivamente el 30 de agosto de 1979 y queda resumido así:

Valor de los

presupuestos

Pesetas

Clases de obra

Número

de obras

704.809.278

Abastecimiento de agua, redes de distri-

bución, saneamiento, vías, instalacio-

nes deportivas, etc. ...............

136

Una vez aprobado el Plan, se inició la tramitación del correspondiente préstamo con el Banco de Crédito Local de España, redacción de proyectos, aportación de documentaciones por los Ayuntamientos y preparación para proceder a las contrataciones, formalizándose en firme 18 obras hasta el 31 de diciembre de 1979. 


\section{AyUda técnica a los Municipios}

Los servicios municipales necesitan de la cooperación de la Diputación no sólo con ejecución de obras o auxilios económicos, sino mediante la orientación económica y técnica e incluso redactando estudios y proyectos, ya que la falta de personal técnico en muchos Ayuntamientos representa un agudo problema que no es fácil superar.

Se redactaron en 1979 proyectos por los distintos grupos de técnicos, clasificados así:

Por ingeniero de caminos, 16 proyectos.

Por ingeniero industrial, 5 proyectos.

Por arquitectos, 14 proyectos.

\section{Caja Provincial de Crédito Municipal}

$\mathrm{Al}$ amparo de lo dispuesto en el apartado 1 del artículo 243 de la Ley de Régimen local, se creó por la Diputación de Cádiz una Caja de Crédito para el mejor desarrollo de la cooperación a los. Municipios.

El capital fundacional fue aportado por la Corporación provincial mediante ingresos anuales, hasta 10 millones de pesetas, incorporando luego a esas cantidades el 15 por 100 de los Planes. bienales de cooperación y los reintegros que realizan los Ayuntamientos por los anticipos reintegrables concedidos en régimen de cooperación.

La finalidad de la Caja Provincial de Crédito Municipal es conceder anticipos a un máximo de cinco años de reintegro, supliendo aportaciones de los Ayuntamientos en las obras incluidas en los Planes de cooperación para necesidades urgentes que se les presenten durante la vigencia de los mismos.

En julio, los reintegros vencidos y no ingresados equivalían a 51.036.364 pesetas, $y$, por ello, realizan un estudio urgente para que no impida el funcionamiento de la Caja.

En noviembre se propusieron al Pleno anticipos por valor de 18.153.054 pesetas.

En diciembre se propusieron al Pleno anticipos por valor de 6.800 .000 pesetas. 


\section{AMPLIACION DEL ABASTECIMIENTO DE AGUAS}

\section{A LA ZONA DE LA BAHIA GADITANA}

La Diputación, consciente, da la solución al problema vital de la ampliación del abastecimiento de aguas a la zona de la bahía gaditana, que comprende todos los Municipios integrados dentro de un semicírculo de 50 kilómetros de radio y centro, Cádiz capital, afectando a unos 600.000 habitantes.

El Ministerio promulgó el Decreto 3138/1972, de 2 de noviembre, en el que reconoce la necesidad de proceder con urgencia a esta ampliación, fijando el presupuesto de las obras a realizar en 1.318 millones de pesetas; el Estado financia un 20 por 100, a fondo perdido, anticipando el 15 por 100 para reintegrar en veinte años, y los Ayuntamientos afectados aportan el 65 por 100 restante, financiando esta operación el Banco de Crédito Local de España.

El importe abonado por el Banco de Crédito Local de España de obras ejecutadas desde 1973 hasta final de 1979 es de 1.450.952.113 pesetas. Por el aumento de los costes de material y mano de obra, la Diputación tuvo que solicitar nuevos préstamos al Banco de Crédito Local de España, y en 1979 figuran en tal concepto 250 millones de pesetas.

\section{HACIENDA PROVINCIAL}

1. PResupuesto ordinario de gastos, ingresos Y LIQUIDACIÓN DEL AÑO 1979

A) Presupuestos gastos (1979) (segundo semestre)

\section{RESUMEN POR CAPITULOS}

\begin{tabular}{|c|c|c|}
\hline Capitulos & Expresion & Pesetas \\
\hline $\begin{array}{l}10^{\circ} \\
20^{\circ} \\
40^{\circ} \\
50^{\circ} \\
6 .^{\circ}\end{array}$ & 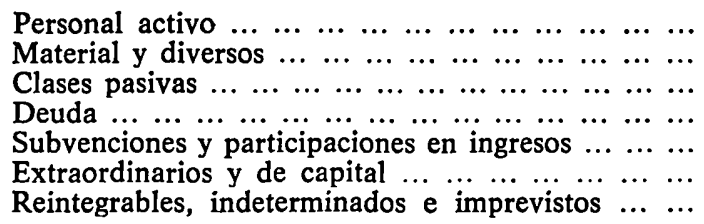 & $\begin{array}{r}603.523 .128 \\
378.905 .531 \\
2.500 .000 \\
106.834 .525 \\
142.500 .000 \\
85.480 .816 \\
80.256 .000\end{array}$ \\
\hline $70^{\circ}$ & Total del estado de gastos $\ldots \ldots \ldots \ldots$ & 1.400 .000 .000 \\
\hline
\end{tabular}


B) Presupuesto ingresos (1979) (segundo semestre)

RESUMEN POR .CAPITULOS

\begin{tabular}{|c|c|c|}
\hline Capitulos & Expresion & Pesetas \\
\hline \multirow[t]{2}{*}{$\begin{array}{l}10^{\circ} \\
20^{\circ} \\
30^{\circ} \\
4 .^{\circ} \\
60^{\circ} \\
70^{\circ}\end{array}$} & 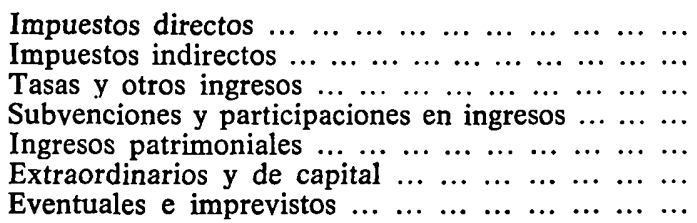 & $\begin{array}{r}48.721 .400 \\
754.635 .589 \\
216.773 .500 \\
187.111 .342 \\
29.138 .100 \\
81.871 .523 \\
81.748 .546\end{array}$ \\
\hline & TOTAL DEL ESTADO DE INGRESOS $\ldots \ldots \ldots \ldots$ & 1.400 .000 .000 \\
\hline
\end{tabular}

C) Liquidación del presupuesto ordinario 1979

I. CREDITOS AUTORIZADOS

Presupuesto ordinario de 1978, prorrogado para $1979 \ldots \ldots \ldots \ldots \ldots \ldots \ldots$

Recursos definitivos

Resultas .......

Presupuesto ordinario de 1978, prorrogado para $1979 \ldots \ldots \ldots \ldots$

Créditos definitivos

$\begin{array}{lllllllllllll}\text { Resultas } & \ldots & \ldots & \ldots & \ldots & \ldots & \ldots & \ldots & \ldots & \ldots & \ldots & \ldots & 430.987 .168\end{array}$

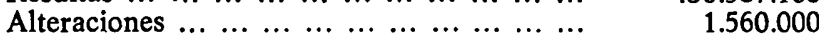

DÉFICIT DEL PRESUPUESTO DEFINITIVO

II. MOVIMIENTO DE FONDOS

Del presupuesto refundido $\ldots \ldots \ldots \ldots$.......

Ingresos

Devoluciones de ingresos 5.627 .359 1.117.741.885

Del presupuesto refundido

1.122 .152 .482

Pagos

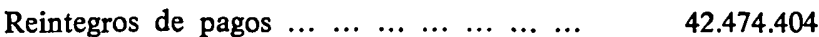
1.079.678.078

EXISTENCIAS EN 30-VI-79, TRASLADADAS AL PRESUPUESTO DE 1979 (SEGUNDO SEMESTRE) 


\section{LIQUIDACION}

Superávit del presupuesto definitivo ......

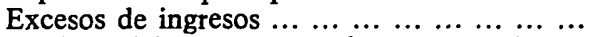

Créditos del presupuesto de gastos no invertidos (economías) $\ldots \ldots \ldots \ldots \ldots$

Déficit del presupuesto definitivo ... ......

Créditos del presupuesto de ingresos no li$\begin{array}{llllllllllll}\text { quidados } & \ldots & \ldots & \ldots & \ldots & \ldots & \ldots & \ldots & \ldots & \ldots & \ldots & \ldots\end{array}$

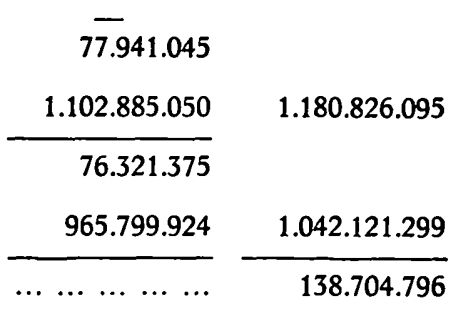

SUPERÁviT

543.743 .269

581.807 .076

supuesto de 1979 (segundo semestre) ...

Créditos pendientes de cobro en dicha fecha.

Obligaciones pendientes de pago en dicha

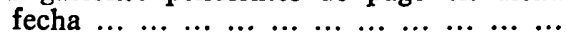

443.102 .280

Diferencia igual a la ANTERIOR

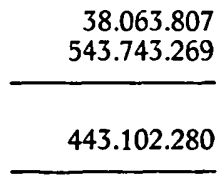

443.102 .280

138.704 .796

\section{LIQUIDACION DEL PRESUPUESTO ORDINARIO 1979}

\section{CREDITOS AUTORIZADOS}

Presupuesto ordinario de 1979 (segundo semestre) $\ldots \ldots \ldots \ldots \ldots \ldots \ldots$

1.400 .000 .000

Recursos definitivos

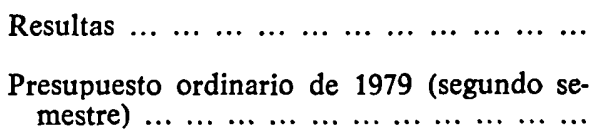

$\frac{581.807 .076}{1.400 .000 .000} \quad 1.981 .807 .076$

Créditos definitivos

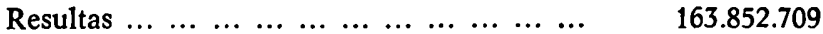

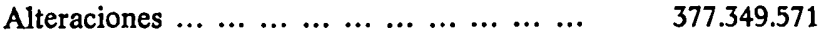

SUPERÁVIT DEL PRESUPUESTO DEFINITIVO $\ldots \ldots \ldots \ldots \ldots \ldots \ldots \ldots$

1.941.202.280

40.604 .796

II. MOVIMIENTO DE FONDOS

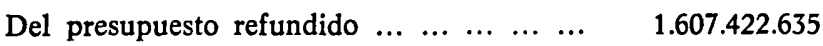

\section{Ingresos}

Devoluciones de ingresos ...............

174.128

1.607.248.507

Del presupuesto refundido

1.450 .623 .323

Pagos

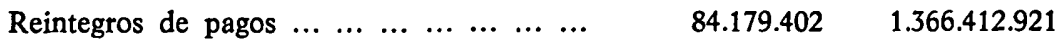

EXISTENCIAS EN 31-XII-79, TRASLADADAS AL PRESUPUESTO DE 1980. 
III. LIQUIDACION

Superávit del presupuesto definitivo ... ...

Excesos de ingresos $\ldots \ldots \ldots \ldots \ldots \ldots$

Créditos del presupuesto de gastos no invertidos (economías) $\ldots \ldots \ldots \ldots \ldots$

Déficit del presupuesto definitivo $\ldots \ldots \ldots$

Créditos del presupuesto de ingresos no liquidados

40.604 .796

118.417 .131

165.562 .886

324.584 .813

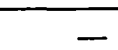

152.848 .393

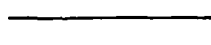

151.848 .393

SUPERÁvit

171.736 .420

IV. COMPROBACION

Existencias en 31-XII-79, trasladadas al presupuesto de $1980 \ldots \ldots \ldots \ldots \ldots \ldots \ldots \ldots$

Créditos pendientes de cobro en dicha fecha.

240.805 .586

340.127 .307

580.932 .893

Obligaciones pendientes de pago en igual fecha

409.196 .473

Diferencia IGUAL A LA ANTERIOR

409.196.473

171.736 .420

2. Presupuesto de gastos e jngresos y liquidación del Servicio DE Recaudación de Contribuciones e Impuestos del Estado. EJERCICIO DE 1979

A) Presupuesto de gastos

RESUMEN POR CAPITULOS

\begin{tabular}{|c|c|c|}
\hline Capítulo & Expresion & Pesetas \\
\hline \multirow[t]{2}{*}{$\begin{array}{l}10^{\circ} \\
20^{\circ} \\
30^{\circ} \\
40^{\circ} \\
50^{\circ} \\
70^{\circ}\end{array}$} & 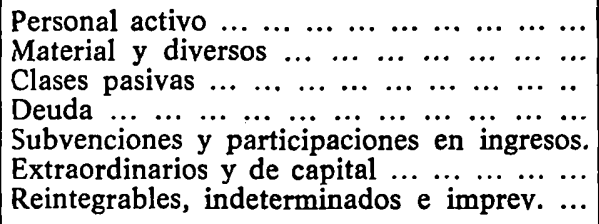 & $\begin{array}{r}66.757 .000 \\
225.000 \\
-\overline{200.000} \\
4.000 .000 \\
1.550 .000 \\
6.373 .000\end{array}$ \\
\hline & Total Estado DE Gastos $\ldots \ldots \ldots \ldots$ & 79.105 .000 \\
\hline
\end{tabular}


B) Presupuesto de ingresos

\begin{tabular}{|c|c|c|}
\hline Capítulo & Expresion & Pesetas \\
\hline \multirow[t]{2}{*}{$\begin{array}{l}10^{\circ} \\
20^{\circ} \\
40^{\circ} \\
50^{\circ} \\
60^{\circ}\end{array}$} & $\begin{array}{llllllll}\text { Impuestos directos } \ldots & \ldots & \ldots & \ldots & \ldots & \ldots & \ldots & \ldots \\
\text { Impuestos indirectos } & \ldots & \ldots & \ldots & \ldots & \ldots & \ldots & \ldots \\
\text { Tasas y otros ingresos } & \ldots & \ldots & \ldots & \ldots & \ldots & \ldots \\
\text { Subvenciones y participaciones } & \text { en } & \text { ingresos } \\
\text { Ingresos patrimoniales } \ldots & \ldots & \ldots & \ldots & \ldots & \ldots & \ldots \\
\text { Extraordinarios y de capital } & \ldots & \ldots & \ldots & \ldots & \ldots \\
\text { Eventuales e imprevistos } & \ldots & \ldots & \ldots & \ldots & \ldots & \ldots\end{array}$ & $\begin{array}{l}\overline{-} \\
72.2 \overline{45} .000 \\
\overline{100.000} \\
\overline{\overline{6}} .000\end{array}$ \\
\hline & TOTAL ESTADO DE INGRESOS $\ldots \ldots \ldots$ & 79.105 .000 \\
\hline
\end{tabular}

C) Liquidación del presupuesto especial de contribuciones 1979

I. CREDITOS AUTORIZADOS

\section{Ingresos}

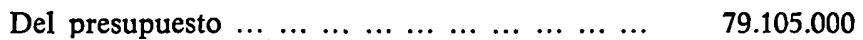

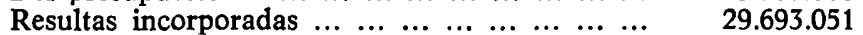

108.798.051

Gastos

Del presupuesto

Resultas incorporadas

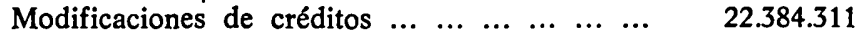

DEFFICIT DEL PRESUPUESTO REFUNDIDO

131.182 .362

22.384.311

II. MOVIMIENTO DE FONDOS

\section{Ingresos}

Del presupuesto

Devoluciones de ingresos

\section{Pagos}

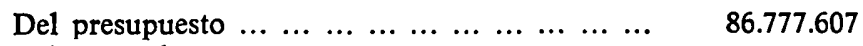

Reintegro de pagos $\ldots \ldots \ldots \ldots \ldots \ldots \ldots \ldots$

Existencia EN CAJA EN 31-XII-1979

\section{LIQUIDACION}

Superávit del presupuesto definitivo

Créditos del presupuesto de gastos no invertidos (economías) $\ldots \ldots \ldots \ldots \ldots$

Excesos de ingresos $\ldots \ldots \ldots \ldots \ldots \ldots \ldots$ 
Déficit del presupuesto definitivo $\ldots . \ldots \ldots \ldots$

Créditos del presupuesto de ingresos no liqui-

22.384.311

dados

IV. COMPROBACION

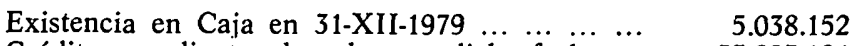

Créditos pendientes de cobro en dicha fecha $\ldots . .33 .893 .124$

38.931 .276

Obligaciones pendientes de pago en dicha fecha.

38.931 .276

38.931 .276

Nota aclaratoria.-Ha producido un beneficio líquido de 11.148 .520 pesetas, y figuran en las resultas de gastos del propio presupuesto, conforme al dictamen emitido por la Comisión Central de Cuentas en su Resolución de 26 de mayo de 1972.

\section{Presupuestos extraordinarios (figuran en la pág. 529).}

4. InVentario de bienes propiedad de la Diputación Provinctal R E S U M E N

\begin{tabular}{|c|c|c|}
\hline & & Pesetas \\
\hline \multirow[t]{2}{*}{$\begin{array}{l}10^{\circ} \\
20^{\circ} \\
30^{\circ} \\
4 .^{\circ} \\
50^{\circ} \\
6.0^{\circ} \\
70^{\circ} \\
8 .\end{array}$} & 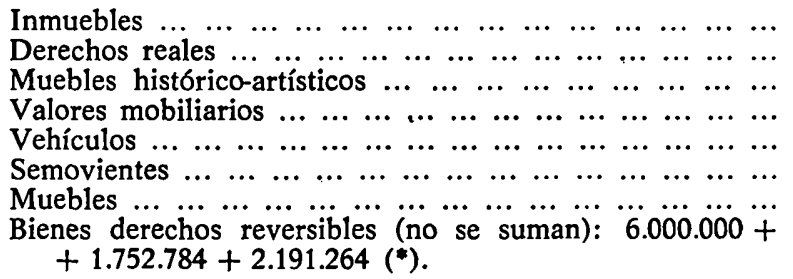 & $\begin{array}{c}1.068 .497 .048,12 \\
- \\
7.636 .550,-\overline{12} \\
12.955 .397,22 \\
7.200 .271,- \\
5.188 .650,- \\
251.791 .260,65\end{array}$ \\
\hline & 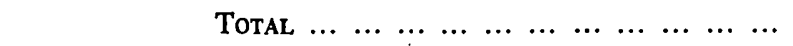 & 1.353.269.176,99 \\
\hline
\end{tabular}

\section{EPILOGO}

Solicitamos de todas las Diputaciones Provinciales las Memorias anuales del ejercicio 1979, si no las han enviado ya, para que así figuren en la Biblioteca del Instituto de Estudios de Administración Local, en beneficio de todas las Corporaciones locales de España, aparte de ser comentadas en esta REvisTa para divulgación y ejemplo de las mismas.

(*) En el Anuario estadístico de las Corporaciones locales 1975. del IEAL, página XLV, exponía, después de una consulta a la Real Academia de la Lengua, que la palabra reversible está admitida en el Diccionario de la Academia de la Lengua y revertible no está admitida. Existe el verbo revertir, de donde se deriva reversible, en el sentido de volver a punto, estado o situación anterior. Existe el verbo revertir, en sentido de verterse, desbordarse algo, que no ha producido, al parecer, ningún derivado en ible. 
DETALLE DEL DESARROLLO ECONOMICO DE LOS PRESUPUESTOS EXTRAORDINARIOS EN VIGOR

(Al 30 de diciembre de 1979)

\begin{tabular}{|c|c|c|c|c|c|}
\hline Número & Concepto & $\begin{array}{c}\text { Presupuesto } \\
\text { Pesetas }\end{array}$ & $\begin{array}{c}\text { Pagos reales } \\
\text { 1970-1979 } \\
- \\
\text { Pesetas }\end{array}$ & $\begin{array}{c}\text { Total } \\
\text { pagado } \\
\text { Pesetas }\end{array}$ & $\begin{array}{c}\text { Falta } \\
\text { por pagar } \\
\text { Pesetas }\end{array}$ \\
\hline \multirow[t]{2}{*}{$\begin{array}{l}3 / 67 \\
3 / 69 \\
3 / 70 \\
4 / 70 \\
5 / 70 \\
1 / 71 \\
2 / 71 \\
2 / 72 \\
1 / 73 \\
5 / 73 \\
6 / 73 \\
3 / 74 \\
1 / 76 \\
3 / 76 \\
4 / 76 \\
1 / 78 \\
2 / 78 \\
1 / 79 \\
2 / 79 \\
3 / 79\end{array}$} & 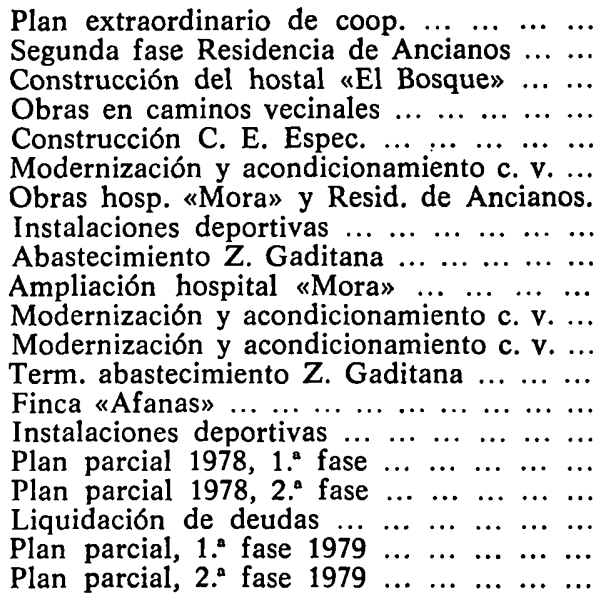 & $\begin{array}{r}84.223 .537 \\
35.875 .888 \\
1.113 .426 \\
99.397 .575 \\
69.335 .497 \\
94.395 .914 \\
60.641 .645 \\
80.200 .000 \\
748.743 .190 \\
54.111 .071 \\
54.893 .602 \\
177.250 .000 \\
800.200 .000 \\
50.050 .000 \\
50.000 .000 \\
318.846 .032 \\
394.863 .535 \\
115.464 .282 \\
195.644 .683 \\
514.055 .039\end{array}$ & $\begin{array}{r}65.670 .702 \\
34.685 .524 \\
3.213 .112 \\
98.871 .192 \\
68.622 .285 \\
94.166 .652 \\
58.013 .563 \\
78.483 .185 \\
746.243 .630 \\
53.208 .955 \\
54.691 .853 \\
176.842 .853 \\
640.770 .396 \\
50.000 .305 \\
18.037 .332 \\
154.361 .214 \\
251.987 .191 \\
115.464 .282 \\
46.882 .200\end{array}$ & $\begin{array}{r}69.064 .818 \\
35.559 .978 \\
3.213 .112 \\
98.871 .192 \\
68.622 .285 \\
94.166 .652 \\
58.013 .563 \\
78.483 .185 \\
746.243 .630 \\
53.208 .955 \\
54.691 .853 \\
176.842 .853 \\
640.770 .396 \\
50.000 .305 \\
18.037 .332 \\
154.361 .214 \\
251.987 .191 \\
115.464 .282 \\
- \\
46.882 .200\end{array}$ & $\begin{array}{r}15.158 .719 \\
315.910 \\
900.314 \\
526.383 \\
713.212 \\
229.262 \\
2.628 .082 \\
1.716 .815 \\
2.499 .566 \\
902.116 \\
201.749 \\
407.147 \\
159.429 .604 \\
49.695 \\
31.962 .668 \\
164.484 .818 \\
142.876 .344 \\
- \\
195.644 .683 \\
467.172 .839\end{array}$ \\
\hline & $\begin{array}{llllllllllllll}\text { Totales } & \ldots & \ldots & \ldots & \ldots & \ldots & \ldots & \ldots & \ldots & \ldots\end{array}$ & 4.002 .304 .916 & 2.810 .216 .426 & 2.814 .484 .996 & 1.187 .819 .920 \\
\hline
\end{tabular}


REVL-1980, núm. 207. RODRIGUEZ SOCORRO, ANTONIO. ACTIVIDADES GENERALES DE LA DIP...

REVL-1980, núm. 207. RODRIGUEZ SOCORRO, ANTONIO. ACTIVIDADES GENERALES DE LA DIP... 


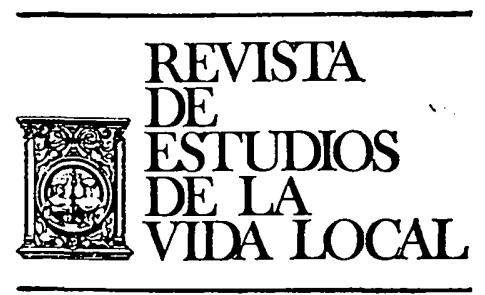

\section{ESTADISTICA}


REVL-1980, núm. 207. RODRIGUEZ SOCORRO, ANTONIO. ACTIVIDADES GENERALES DE LA DIP...

REVL-1980, núm. 207. RODRIGUEZ SOCORRO, ANTONIO. ACTIVIDADES GENERALES DE LA DIP... 